\title{
Synthesis of Some New Nucleoside Analogues Containing Seven Membered Ring and Studying Their Biological Activity
}

\section{Thanaa Mahdi Al-Mouamin Omar Abdulateef Mohammed}

\author{
SurorAbdulrahman Mahdi \\ Yasmeen H. Muhamad
}

Department of Chemistry, College of Science, University of Baghdad, Al-Jaderia, Baghdad-Iraq

E-mail:surchem_82@yahoo.com

Received 17/5/2015

Accepted 20/12/2015

(1) $(9)$

This work is licensed under a Creative Commons Attribution-NonCommercialNoDerivatives 4.0 International Licens

\begin{abstract}
:
In this work, a series of new Nucleoside analogues (D-galactopyranose linked to oxepanebenzimidazole moiety) was synthesized via multisteps synthesis.

The first step involved preparation of two benzimidazoles 2styrylbenzimidazole and 2-(phenyl ethynyl) benzimidazole via reaction of phenylenediamine with cinnamic acid or $\beta$-phenyl propiolic acid. Electrophilic addition of the prepared benzimidazoles by three anhydrides in the second step afforded (4-6) and (14-16) which in turn were treated with 1,2,3,4-di-Oisopropylidene galactopyranose in the third step to afford a series of the desirable protected nucleoside analogues (7-9) ,(17-19)which after hydrolysis in methanolic sodium methoxidein the fourth step afforded the free nucleoside analogues (10-12) and (20-22) .The synthesized compounds were identified by FT-IR and some of them by ${ }^{1} \mathrm{H}-\mathrm{NMR}$ and ${ }^{13} \mathrm{C}-\mathrm{NMR}$.

The synthesized oxepane nucleoside analogues were screened for their antibacterial activity against three types of bacteria including Staphylococcusaureus ,Bacillus(gram positive) andE.coli (gram negative) bacteria repectively.
\end{abstract}

Key words: Nucleoside, oxepane nucleoside, isopropyledenegalactopyranose.

\section{Introduction:}

Oxepane diones that are heterocyclic compounds with seven-membered oxycyclic are found in a number of natural products such as zoapatanol ,regiolenyne and breveral [1]. Nowaday they have been receiving more attention for their wide range of biomedical applications as cytotoxicity against cancer cell lines, antimicrobial activity and inhibition of several enzyme activities [2]. Also they applied as achemotherapeutic used to treat advanced brain tumors[3],[4].

On the other hand, benzimidazole has an important chemical entity in pharmaceuticals [5]. Benzimidazole derivatives possess antibacterial effects, antifungal activity, HIV inhibitors, 
antiviral effects, antihypertensive agents, antiulcer activity, antiproliferative activity, antitumor activity, antioxidant agents[6].In another approach nucleoside analogues have found to be important moiety in creation of novel medical compounds. Even though, they have been studied a long time ago, there is still a great potential development of new therapeutic agents as antiviral compounds to treat diseases caused by HIV and hepatitis [7], antimicrobial agents [8], anticancer agents [9], [10].These observations inspire us to synthesis new nucleoside analogues containing oxepane and bezimidazole as a anucleobase and galactose as a asugar moiety which shows good biological activities against three types of bacteria.

\section{Materials and Methods:}

Melting points were recorded using Gallenkhamp electro thermal melting point apparatus. FT-IR spectra were recorded on SHIMADZU FT-IR 8400 fourier transform intrared spectrophotometer using $\mathrm{KBr}$ disc or thin films. 'H-NMR spectra were recorded on Brukerspectrospin ultra shield magnet $300 \mathrm{MHz}$ instrument using $\mathrm{Me}_{4} \mathrm{Si}$ as the internal standard and DMSO-d6 as solvent.TLC plates were used with an aluminum backing $\left(0.2 \mathrm{~mm}, 60 \mathrm{~F}_{254}\right)$, the reactions were monitored by TLC and visualized by development of the TLC plates. Incubator Heraeus D-63450 (Germany) model was used for incubation samples for biological study.

\section{1- Synthesis of 1,2:3,4-di-O-} isopropylidene-D-galacto pyranose (1)

Zinc chloride $(8.87 \mathrm{~g}, 88 \mathrm{mmol})$ was partially dissolved in $(125 \mathrm{ml})$ acetone and $(0.4 \mathrm{ml})$ concentrated sulfuric acid was added at room temperature to give a clear solution. $(10 \mathrm{~g}, \quad 56 \mathrm{mmol})$ D- galactosewas added in one portion and the resulting white suspension was stirred for 6 hours at room temperature. Asuspension of $(20 \mathrm{~g}, 189 \mathrm{mmol})$ sodium carbonate in $(30 \mathrm{ml})$ water added to the yellow reaction mixture at $0^{\circ} \mathrm{C}$ in medium sized portions. The suspension was allowed to stir for $30 \mathrm{~min}$ before filtration and solvent removal in vacuo to give the crude product as tallow oil below the aqueous layer. The organic fraction was separated from the aqueous layer, followed by further extraction with $(3 \times 50 \mathrm{ml})$ diethylether. The organic layer was dried over sodium sulfate [11], and the solvent removed in vacuo to yield $1,2: 3,4-d i$-O-iso propylidene-Dgalactso (1) as a pale yellow syrup (87\%), Rf $=0.23\left(\mathrm{Et}_{2} \mathrm{O}\right)$.

2- Synthesis of 6-Bromo-1,2:3,4$\mathrm{di}-\mathrm{O}$-isopropylidene-D-galacto pyransoe(2)

The $\operatorname{sugar}(1)(20 \mathrm{~g}, 76.9 \mathrm{mmol})$ was treated with $50 \%(\mathrm{w} / \mathrm{v})$ hydrogen bromide in acetic acid $(52.5 \mathrm{ml})$. The solution was kept in $0^{\circ} \mathrm{C}$ unitl TLC in indicated reaction completion (generally within one hour), then poured into an ice-cold chloroform $(35 \mathrm{ml})$, washed with iced water $(3 \times 25 \mathrm{ml})$ and then with saturated aqueous solution of sodium bicarbonate to remove the remaining acid [12] . After a find wash with iced water $(25 \mathrm{ml})$ the organic phase was dried over anhydrous sodium sulphate to give (2) as syrup (43\%) the isolated sugar bromide (2) was used directly for the nucleoside synthesis : Rf $0.43\left(\mathrm{Et}_{2} \mathrm{O}\right.$ : EtOH 1:1), yield $43 \%$.

\section{3- Synthesis of 2-Styryl benzimidazole (3)}

Cinnamic acid $(2.96 \mathrm{~g}, 20 \mathrm{mmol})$ was dissolved in $(25 \mathrm{ml})$ absolute ethanol, $O$ phenylenediamine $(2.16 \mathrm{~g}, 20 \mathrm{mmol})$ was added. The mixturewas refluxed for $12 \mathrm{~h}$ using water bath. then cooled and poured onto $(50 \mathrm{ml})$ ice-cold water 
containing $(1 \mathrm{ml})$ of concentrated $\mathrm{HCl}$,scratched for $15 \mathrm{~min}$, filtered [13][14].Dried and recrystallized from ethanol to give (2) as yellow precipitate, yield $75 \%$.

\section{4- Synthesis of benzimidazol-2-yl oxepane-4,7-diones (4-6)}

compound (3) (1 mmol) was placed in round bottomed flask; (succinic ,phthalicor maleic) anhydride (1 mmol) in $(10 \mathrm{ml})$ benzene wasadded,then refluxed on water bath for 1 hour the solvent was evaporated and the solid precipitated was recrystalized from tetrahydro furan (THF). Physical properties of compounds (4-6) are listed in Table (2).

\section{5-Synthesis of 6-(N-(phenyl oxepane $\quad-4,7-$ dione $\quad-3-y 1)$ benzimidazolyl)- $\quad 1,2,3,-d i-O$ - isopropylidene galactopyranoses (7-9)}

$(1.08 \mathrm{mmol})$ isopropylidene sugar bromide (2) was dissolved in $(10 \mathrm{ml})$ of dried xylene, then $(0.533 \mathrm{mmol})$ benzimidazolyl derivatives (4-6) were added and refluxed with vigorous stirring for one hour [12]. TLC (chloroform ether 9:1), the result mixture was cooled to room temperature then filtred and washed with $(5 \mathrm{ml})$ ethanol then dried to give after silica gel column chromatography the protected nucleoside [12]. Physical properties of componneds (7-9) are listed in Table (3).

\section{6-Synthesis of 6-(N-(phenyl oxepane $\quad-4,7-$ dione $\quad-3-y 1)$ benzimidazoyl) galactopyranoses (10-12)}

A solution of $(0.292 \mathrm{mmol})$ of the protected nucleoside (7-9) in (7 ml) of $0.1 \mathrm{M}$ methanolic sodium methoxide was refluxed with stirring for half hour TLC (chloro form : ethanol 8:2) showed that the reaction was complete. The mixture was neutralized with acetic acid and evaporated to dryness. The residue was partitioned between water and chloroform and the aqueous phase was evaporated to dryness in vacuo. The reside was dissolved in methanol $(3 \mathrm{ml})$ and then chromatographed on a column of silicagel using 9:1 chloroform methanol as developer [12]. Afforded free nucleoside analogueswas purified by recrystalization from ethanol-ether. Physical properties of compounds (1012) are listed in Table (4).

\section{7- Synthesis of 2-(phenyl ethynyl) benzimidazole (13)}

The titled compound was prepared by following the same procedure used in preparation of 2cinnamoylbenzimidazole except using of $\beta$-phenyl propiolic acid instead of cinnamic acid. The product was purified by recrystallization from elhanol yield $73 \%$.

\section{8- Synthesis of benzimidazolyl - 2,3-didehydo oxepane -4,7- diones (14-16).}

The titled compounds were prepared following the same procedure used in the preparations of compounds (4-6) except using of 2-(phenyl ethynyl) instead of 2-styryl benzimidazole. The final products was purified by recrystallization from tetrahydrofuran (THF) physical properties of compounds (14-16) are listed in Table(9).

9- Synthesis of 6-(N-(phenyl -2,3didehdro oxepane $-4,7$ dione -3 yl) benzimidazolyl) - 1,2,3,4-di$O$-isopropylidene galactopyranoses (17-19).

The titled compounds were prepared by following the same procedure used in the preparations of compounds (7-9) except using of benzimidazolyloxcpane 
-4,7-dions(14-16) instead of compounds (4-6). The final products were purified by recrystallization from chloroform physical properties of compounds (1719) and they are listed in Table (10).

\section{0- Synthesis of 6-(N-(phenyl -} 2,3-didehydro oxepane -4,7dione -3-yl) benzimidazolyl) galactopyranoses(20-22)

The titled compounds were prepared following the same procedure used in the preparation of compounds (10-12) using of protected nucleosides (1719)instead of (7-9). The product was recrystallized from ethanol ether. Physical properties of compounds (2022) were listed in Table (11).

\section{1- Microbiological test:}

Nutrient agar was added to (1L) of distilled water in suitable conical flask with stirring and heating until complete dissolving then the flask was stoppered by cotton and the medium was sterilized in an autoclave for 20 minutes at $\left(121^{\circ}\right)$ under pressure at 15 pound/inch. The medium was placed in petridishes about $(20 \mathrm{ml})$ for each one and was left to cool and solidified. The studied bacteria and fungi were placed on the nutrient agar surface using the loop and by streaking processor then the discs saturated with tested compound solutions. The samples were incubated for 24 hours at $37^{\circ} \mathrm{C}$ [15].

\section{Results and discussion}

The synthetic route was started with 2- styrylbenzimidazole (3) and 2(phenyl ethynyl) benzimidazole (13) which were synthesized from condensation reaction of phenylenediamine withequimolar quantity of cinnamic acid or $\beta$ - Phenyl propiolic acid respectively via Debus Radziszewski, reaction.

Thesetwocompoundswere reacted with different acid anhydrides (succinic, phthalic, and maleic) to give new substituted oxepanedione derivatives (46) and (14-16) subjected to nucleophilic substitution reaction with bromo sugar (2)to give new protected nucleoside analogues (7-9) and (17-19) respectively to obtain our synthetic goal. The free nucleoside analogues play an important role in treatment of tumor cell and as antiviral drugs.Benzimidazole and oxepane (oxepine) ring also have abroad spectrum of biological activities. The overall work steps show in Scheme (1) and Scheme (2). 

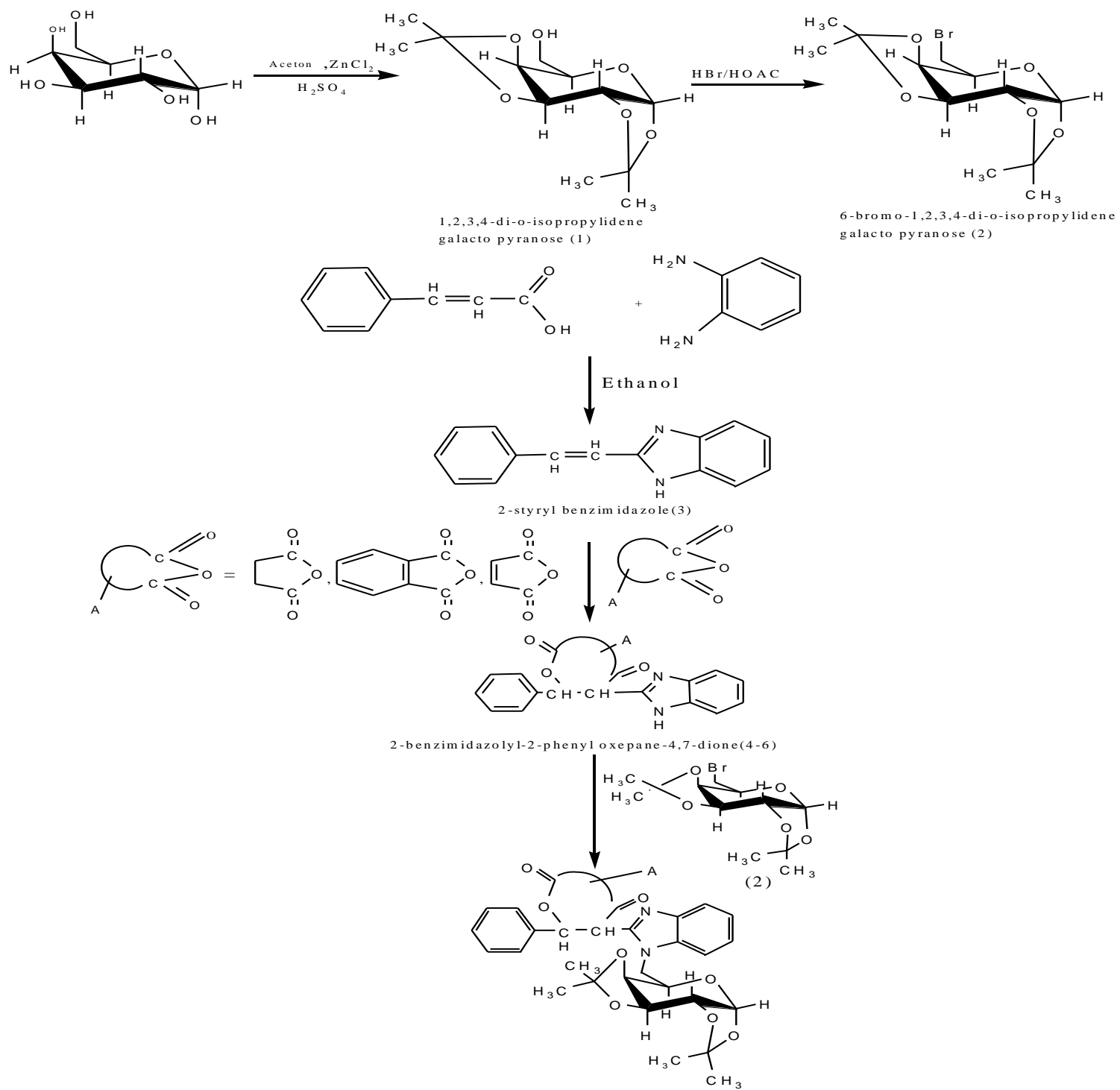

6-(N-(phenyl oxepane-4,7-dione-3-yl)benzim idazoly1)-1,2,3,4-di-o-isopropylidene galacto pyranose (7-9).

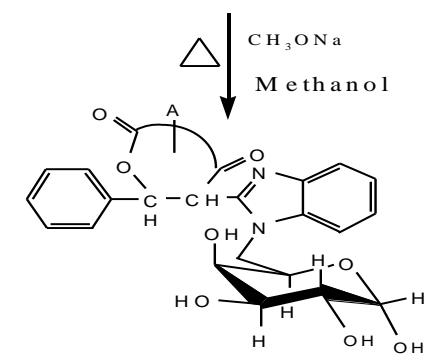

Scheme (1):Synthesis of 6-(N-(phenyl oxepane -4,7-dione -3-yl) benzimidazoyl) galactopyranoses (10-12) 


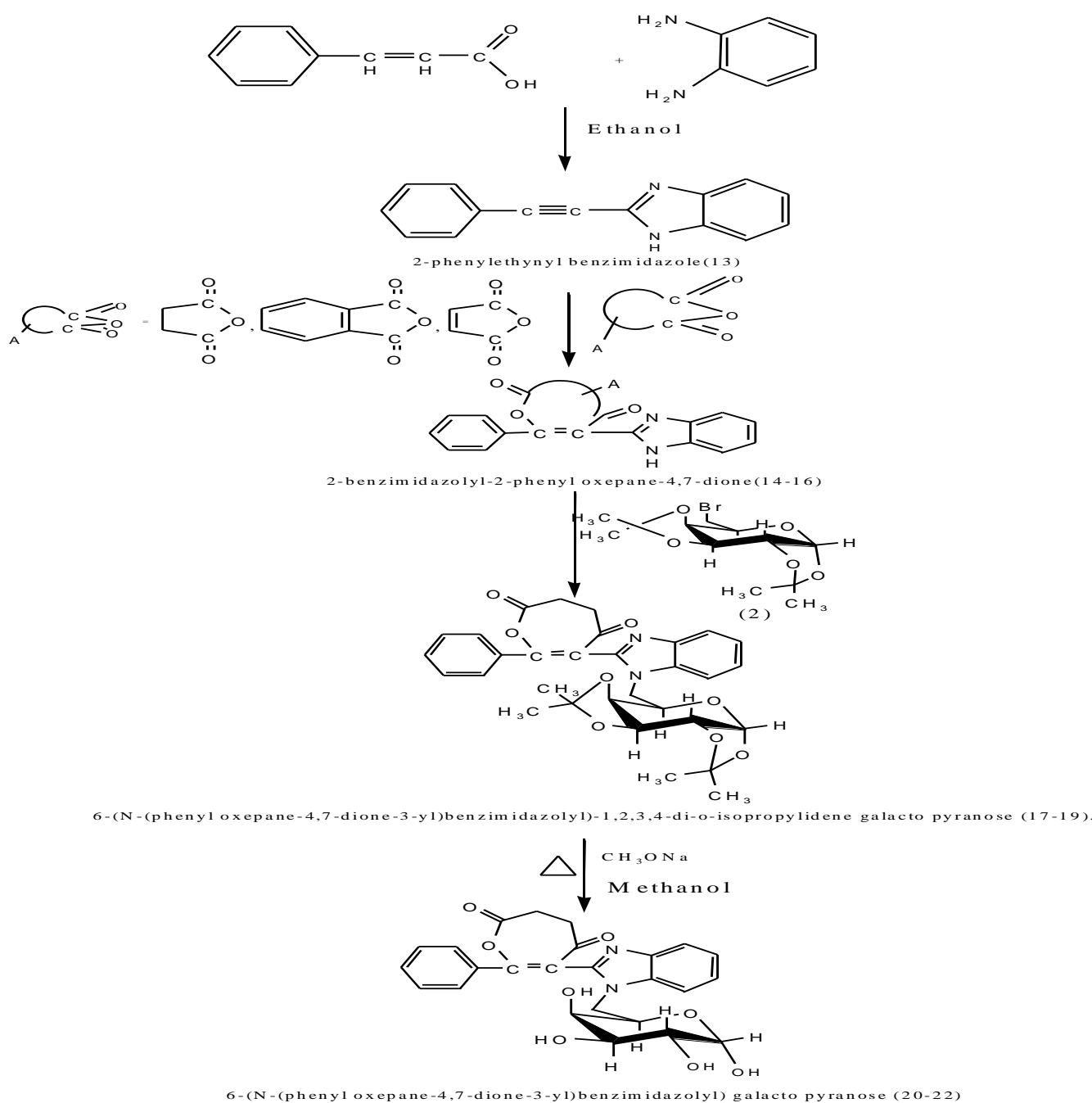

Scheme (2) :Synthesis of 6-(N-(phenyl -2,3-didehydro oxepane -4,7-dione -3-yl) benzimidazolyl) galactopyranoses $(20-22)$

The reaction of D- galactose with aceton in presence of anhydrous zinc chloride and sulfuric acid afforded $1,2: 3,4,-d i-$ $O$ - isopropylidene - D- galactopyranose (1) in high yield. FT- IR spectrum of (1) showed absorption band at $(3458) \mathrm{cm}^{-1}$ assigned to $v(\mathrm{O}-\mathrm{H})$ while this band was disappeared in FT- IR spectrum of 6Bromo- $1,2: 3,4 \quad-\quad d i \quad-\quad O-$ isopropylidenegalactopyranose (2) with an absorption band at (698) $\mathrm{cm}^{-1}$ due to $v(\mathrm{C}-\mathrm{Br})$ group [16] ${ }^{1} \mathrm{H}-\mathrm{NMR}$ spectrum of compound (2) showed two singlet signals at $(\delta=0.9$ and 1.1) ppm belong to protons of four methyl group ,singlet signal at $(\delta=2.5)$ ppm belong to $(\mathrm{CH}-$ Br)and signals at $(\delta=3.3-4)$ ppm belong to $(\mathrm{CH}-\mathrm{OR})$ proton,${ }^{13} \mathrm{C}-\mathrm{NMR}$ spectrum showed signals at $(\delta=39.1-39.9) \mathrm{ppm}$ belong to carbon of four methyl, signals at $(\delta=61.6) \mathrm{ppm}$ and $(\delta=65.9) \mathrm{ppm}$ belong to $\left(\mathrm{CH}_{2}-\mathrm{OR}\right)$ and signal at $(\delta=$ 40.2)ppm belong to $\left(\mathrm{CH}_{2}-\mathrm{Br}\right)$. This indicated the formation 6- bromo 1,2,3,4 - $d i$ - $O$ isopropylidenegalactopyranose (2). On the other hand two $\alpha, \beta$-unsaturated carboxylic acids named cinnamic acid and $\beta$ - phenyl Propiolic acid were condensed with $O$ - Phenylenediamine to afford 2- styrylbenzimidazole (3) and 
2- ( $\beta$ - phenyl ethynyl) benzimidazole (13) in the first step. FT - IR spectra of (3) and (13) showed appearance of several bands, $v(\mathrm{C}=\mathrm{N})$ benzimidazole,$v$ $(\mathrm{C}-\mathrm{N})$ benzimidazol and $v(\mathrm{~N}-\mathrm{H})$ benzimidazol at , $(1589-1577) \mathrm{cm}^{-1}$ $(1313-1319) \mathrm{cm}^{-1}$ and $(3325-3444) \mathrm{cm}^{-1}$ respectively, and absence of $v(\mathrm{O}-\mathrm{H})$. ${ }^{1} \mathrm{H}-\mathrm{NMR}$ spectrum of compound (3) showed signal at $(\delta=2.3) \mathrm{ppm}$ belong to $(\mathrm{N}-\mathrm{H})$ proton ,douplet signal at $(\delta=$ $6.2,6.3) \mathrm{ppm}$ belong to $(\mathrm{CH}=\mathrm{CH})$ vinylic protons and multiplet signals at $(\delta=$ 7.03-7.48)ppm belong to aromatic protons $\quad .{ }^{13} \mathrm{C}-\mathrm{NMR}$ spectrum of compound (3) showed signal at $(\delta=$ 39.97)ppm belong to ( $\underline{\mathrm{C}}-\mathrm{NH})$ carbon ,two signals at $(\delta=114.2,119.1) \mathrm{ppm}$ belong to $(\mathrm{C}=\mathrm{C})$ vinylic carbon, signals at $(\delta=122.44-143.89) p p m$ belong to (C-C) aromatic carbon and signal at $(\delta=$ 168)ppm belong to $(\mathrm{C}=\mathrm{N})$ carbon.

The second step involved synthesis of oxepanedionebenzimidazole (4-6) and 2,3 ,

didehydrooxepanedionebenzimidazole (14-16) via reaction of acid anhydride (succinic, phthalicand maleic anhydride) with Compound 2- styrylbenzimidazole (3) or $2-(\beta-$ phenyl ethynyl) benzimidazole (13). FT - IR spectra of (4-6) and (14-16) gave absorption bands at $(1685-1693) \mathrm{cm}^{-1},(1700-1733) \mathrm{cm}^{-1}$ attributed to $v(\mathrm{C}=\mathrm{O})$ ketone and $v$ $(\mathrm{C}=\mathrm{O})$ ester respectively; besides the absence of $v(\mathrm{C}=\mathrm{O})$ anhydride at $(1800$ 1840) $\mathrm{cm}^{-1}$ indicated the success of addition reaction of anhydride (4-6) and (14-16) which subjected to anucleophilic substitution by treating with 6 - bromo- 1,2,3,4- di-Oisopropylidene - D - galactopyranose (2) in the third step to afford $6-(\mathrm{N}-$ (phenyl oxepane $-4,7-$ dione $-3-\mathrm{yl}$ ) benzimidazolyl) - 1,2,3,4. $\mathrm{Di}-\mathrm{O}-$ isoproplidenegalactopyaroses (7-9) and 6- $\quad(\mathrm{N}-$ (phenyl - 2,3 didehydrooxepane - 4,7 - dione - 3- yl) benzimidazole) $-1,2,3,4-d i-O-$ isopropylidenegalactopyranoses (17-19).
TheFT-IR spectrum showed disappearance of bands $v(\mathrm{C}-\mathrm{Br}), v(\mathrm{~N}$ $\mathrm{H})$ benzimidazole with appearance of bands at $(1271-1342) \mathrm{cm}^{-1}$ attributed to $v(\mathrm{C}-\mathrm{N})$ nucleoside. ${ }^{1} \mathrm{H}-\mathrm{NMR}$ spectrum of compound (5), showedsignal at $(\delta=2.3)$ ppm belongs to $(\mathrm{N}-\mathrm{H})$ proton, signal at $(\delta=2.74) \mathrm{ppm}$ belongs to $(\mathrm{CH}-$ $\mathrm{C}=\mathrm{O})$ proton, signal at $(\delta=3.6) \mathrm{ppm}$ belongs to $(\mathrm{CH}-\mathrm{O}-\mathrm{C}=\mathrm{O})$ proton, multiplet signals at $(\delta=6.5-7.9) \mathrm{ppm}$ belong to aromatic protons,${ }^{13} \mathrm{C}-\mathrm{NMR}$ showed singletsignal at $(\delta=28.74) \mathrm{ppm}$ belongs to $(\underline{\mathrm{C}} \mathrm{H}-\mathrm{C}=\mathrm{O})$ carbon ,signal at $(\delta=39.9) \mathrm{ppm}$ belongs to $(\underline{\mathrm{CH}}-\underline{\mathrm{CH}})$ carbon ,multiplet signals at $(\delta=128.12$ 143.87)ppm belong to aromatic carbon and signal at $(\delta=173.56) \mathrm{ppm}$ belong to $(\mathrm{C}=\mathrm{N})$ carbon.

For synthesis of 6- (N - (phenyl oxepane $-4,7$ - dione $-3-\mathrm{yl}$ ) benzimidazolyl) galactoPyranoses (1012) and 6- (N- (phenyl - 2,3 - didehydro - oxepane - 4,7- dione - 3- yl) benzimidazolyl) galactopyranoses (20$22)$ in the fourth step , (7-9) and (17-19) were allowed to hydrolize by methanolic Sodium methoxide at reflux temperature . FT - IR spectrum of (10-12) and (2022) showed appearance of absorption band at (3350-3357) $\mathrm{cm}^{-1}$ assigned to $v$ $(\mathrm{O}-\mathrm{H})$ glycolic and absence of $v(\mathrm{C}-\mathrm{O}-$ C) ether, other absorption bands to (1012) and (20-22)

Were (1340) cm $\mathrm{cm}^{-1},(1579-1580) \mathrm{cm}^{-1}$, $(1280-1311) \mathrm{cm}^{-1}, \quad(1695-1716) \mathrm{cm}^{-1} \mathrm{due}$ tov $(\mathrm{C}-\mathrm{N})$ nucleoside, $v(\mathrm{C}=\mathrm{N})$ imidazole ,$v(\mathrm{C}-\mathrm{N})$ imidazole,$v \quad(\mathrm{C}=\mathrm{O})$ oxepane respectively. Other details of FT- IR spectral data of the prepared mentioned compounds are listed in Tables $(5,6,7,8)$ and $(12,13,14)$. On the other hand structures of Compound (11) were confirmed also by ${ }^{1} \mathrm{H}$ - NMR spectrum which showed signal at $(\delta=1.9) \mathrm{ppm}$ belong to $\left(\mathrm{CH}_{2}-\mathrm{NR}_{2}\right)$ proton signal at $(\delta=2.5) \mathrm{ppm}$ belong to $(\underline{\mathrm{C}} \mathrm{H}-\mathrm{C}=\mathrm{O})$ proton, signal at $(\delta=3.6) \mathrm{ppm}$ belong to $(\underline{\mathrm{C}} \mathrm{H}-\mathrm{O}-\mathrm{C}=\mathrm{O})$ proton. 
${ }^{13} \mathrm{C}$-NMRspectrum of compound (11) showed signal at $(\delta=28.23)$ ppm belongs to $(\underline{\mathrm{C}} \mathrm{H}-\mathrm{C}=\mathrm{O})$ carbon, signal at $(\delta=$ 38.91)ppm belongs to $\left(\mathrm{CH}_{2}-\mathrm{NR}_{2}\right)$ carbon and signal at $(\delta=67.40) \mathrm{ppm}$ belongs to $(\underline{\mathrm{CH}}-\mathrm{O}-\mathrm{C}=\mathrm{O})$ carbon.

\section{Microbiological test}

The prepared nucleosides are screened for their antibacterial activity against three types of bacteria Bacillu, Staphylococcusaureus (gram positive) and E.coli(gram negative) . They showed different biological activities against these bacteria as shown in Table (15). The result showed a high antibacterial activity of the prepared nucleosidesagaist gram negative more than gram positive, thus compounds (712),(17-22) showed highantibacterial activity against E.coli. Also antibacterial activity of the prepared nucleosides depends on nature of substituents in their molecules, thus compounds (17),(18) and (20) which are substituted phenyl ethynyl showed a high antibacterial activity against Staphylococcus aureusalso compounds (8) and (19) have a high antibacterial activity against this bacteria. Other compounds showed a moderate activity against this bacteria. Finally, compounds (7),(18) and(21) showed a high antibacterial activity against Bacillus,compounds (9), (20) and(22) showed no activity against this bacteria; other compounds showed a moderate activity against this bacteria.

Table (15) inhibition zones to compounds (7-12), (17-22) in $\mathrm{mm}$

\begin{tabular}{|c|c|c|c|}
\hline \multirow{2}{*}{$\begin{array}{c}\text { Comp. } \\
\text { No. }\end{array}$} & $\begin{array}{c}\text { Gram } \\
\text { negative }\end{array}$ & \multicolumn{2}{|c|}{ Gram positive } \\
\cline { 2 - 4 } & $\underline{\text { E.Coli }}$ & $\frac{\text { Bacillus }}{\text { Staphylococcus }}$ & $\frac{\text { aureus }}{11}$ \\
\hline 7 & 14 & 12 & 12 \\
\hline 8 & 16 & 10 & 11 \\
\hline 9 & 15 & N & 11 \\
\hline 10 & 14 & 7 & 11 \\
\hline 11 & 16 & 11 & 13 \\
\hline 12 & 12 & 11 & 15 \\
\hline 17 & 16 & 11 & 14 \\
\hline 18 & 15 & 14 & 12 \\
\hline 19 & 15 & 11 & 13 \\
\hline 20 & 11 & N & 11 \\
\hline 21 & 13 & 12 & 11 \\
\hline 22 & 12 & N & r \\
\hline
\end{tabular}

$\mathrm{N}=$ No inhibition, Inhibition zone (5-7) $\mathrm{mm}=$ Slightly active, Inhibition zone (8-11) $\mathrm{mm}=$ Moderately active, Inhibition zone $>12 \mathrm{~mm}$ Highly active

Table (1) physical properties of the prepared compounds (1), (2), (3), (13).

\begin{tabular}{|l|c|c|c|c|c|}
\hline $\begin{array}{c}\text { Comp. } \\
\text { No. }\end{array}$ & Compound Structure & Color & Rf solvent system & Yield \% & m.p C \\
\hline 1 & $\begin{array}{c}\text { Pale yellow } \\
\text { syrup }\end{array}$ & $\begin{array}{c}\text { Diethyl ether- } \\
\text { ethanol }\end{array}$ & 87 & $\begin{array}{c}\text { b.p= } \\
119\end{array}$ \\
\hline 2 & $\begin{array}{c}\text { Brown } \\
\text { syrup }\end{array}$ & $\begin{array}{c}\text { Diethyl ether- } \\
\text { ethanol }\end{array}$ & 43 & 104 \\
\hline 13 & Pale yellow & Ethanol-acetone & 77 & $92-94$ \\
\hline
\end{tabular}


Table (2) physical properties of Substituted oxepane 4,7-diones (4-6).

\begin{tabular}{|c|c|c|c|c|c|}
\hline $\begin{array}{c}\text { Comp. } \\
\text { No. }\end{array}$ & Compound Structure & Color & $\begin{array}{c}\text { Yield } \\
\%\end{array}$ & m.p $\mathrm{C}^{0}$ & $\begin{array}{c}\begin{array}{c}\text { Recrystallization } \\
\text { solvent }\end{array} \\
\end{array}$ \\
\hline 4 & & $\begin{array}{c}\text { Deep } \\
\text { brown }\end{array}$ & 70 & $82-84$ & THF \\
\hline 5 & & $\begin{array}{l}\text { Grayish- } \\
\text { white }\end{array}$ & 73 & $173-176$ & THF \\
\hline 6 & & Off white & 66 & $126-128$ & THF \\
\hline
\end{tabular}

Table (3) physical properties of oxepane benzimidazole isopropylidene- D galactopyranose $(\mathbf{7 - 9})$.

\begin{tabular}{|l|l|l|l|l|l|}
\hline $\begin{array}{c}\text { Comp. } \\
\text { No. }\end{array}$ & Color & $\begin{array}{c}\text { Yield } \\
\%\end{array}$ & m.p C $^{\mathbf{0}}$ & $\begin{array}{c}\text { Recrystallization } \\
\text { Solvent }\end{array}$ \\
\hline 7 & Compound Structure & Chloroform & Dark \\
brown
\end{tabular}

Table (4) physical properties of oxepane benzimidazole $\beta$ - D - galactopyranose (10-12)

\begin{tabular}{|c|c|c|c|c|c|}
\hline $\begin{array}{c}\text { Comp. } \\
\text { No. } \\
\end{array}$ & Compound Structure & Color & $\begin{array}{c}\text { Yield } \\
\%\end{array}$ & m.p $\mathrm{C}^{0}$ & Recrystallization solvent \\
\hline 10 & & $\begin{array}{c}\text { Pale } \\
\text { yellow }\end{array}$ & 75 & $240 \mathrm{dec}$ & $\begin{array}{c}\text { Ethanol- ether } \\
(1: 1)\end{array}$ \\
\hline 11 & & $\begin{array}{c}\text { Pale } \\
\text { yellow }\end{array}$ & 82 & $225 \mathrm{dec}$ & $\begin{array}{c}\text { Ethanol- ether } \\
(1: 1)\end{array}$ \\
\hline 12 & & $\begin{array}{c}\text { Pale } \\
\text { yellow }\end{array}$ & 78 & $230 \mathrm{dec}$ & $\begin{array}{c}\text { Ethanol- ether } \\
(1: 1)\end{array}$ \\
\hline
\end{tabular}


Table (5) FT-IR spectral data $\left(\mathrm{cm}^{-1}\right)$ of compounds (1), (2), (3), (13).

\begin{tabular}{|c|c|c|c|c|c|c|}
\hline $\begin{array}{l}\text { Comp. } \\
\text { No. }\end{array}$ & Compound Structure & $v(\mathrm{O}-\mathrm{H})$ & $v(C-O-C)$ & $\begin{array}{l}v(\mathrm{C}-\mathrm{O}) \\
\text { alcohol } \\
\end{array}$ & $v(\mathrm{C}-\mathrm{H})$ & $v(C-C)$ \\
\hline 1 & & 3458 & 1166 & 1070 & 2977 & 1002 \\
\hline \multirow{2}{*}{2} & & $v(\mathrm{C}-\mathrm{Br})$ & $v(C-O-C)$ & $v(\mathrm{C}-\mathrm{O})$ & \multirow{2}{*}{2925} & \multirow{2}{*}{1041} \\
\hline & & 698 & 1122 & 1072 & & \\
\hline \multirow[t]{2}{*}{3} & & $v(\mathrm{~N}-\mathrm{H})$ & $\begin{array}{l}v(\mathrm{C}=\mathrm{C}) \\
\text { vinylic }\end{array}$ & $\begin{array}{c}v(C=C) \\
\text { aromatic } \\
v(C=N) \\
\text { imidazole }\end{array}$ & $\begin{array}{c}v(\mathrm{C}-\mathrm{N}) \\
\text { imidazole }\end{array}$ & $\begin{array}{c}v(\mathrm{C}-\mathrm{H}) \\
\text { aromatic }\end{array}$ \\
\hline & & 3325 & 1629 & 1589 & $\begin{array}{l}1286 \\
1313 \\
\end{array}$ & 3066 \\
\hline \multirow[t]{2}{*}{13} & & 3444 & $\begin{array}{c}\mathbf{v}(\mathbf{C} \equiv \mathbf{C}) \\
\text { alkyne }\end{array}$ & \multirow{2}{*}{$\begin{array}{l}1635 \\
1577\end{array}$} & \multirow[t]{2}{*}{1319} & \multirow[t]{2}{*}{3056} \\
\hline & & & 2115 & & & \\
\hline
\end{tabular}

Table (6) FT-IR spectral data $\left(\mathrm{cm}^{-1}\right)$ of subistituted oxepane-4,7-dione (4-6)

\begin{tabular}{|c|c|c|c|c|c|c|c|}
\hline $\begin{array}{l}\text { Comp. } \\
\text { No. }\end{array}$ & Compound Structure & $\begin{array}{l}v(\mathrm{C}=\mathrm{O}) \\
\text { ketone }\end{array}$ & $\begin{array}{c}v(\mathrm{~N}-\mathrm{H}) \\
\text { imidazole }\end{array}$ & $\begin{array}{c}v(C=C) \\
\text { Aromatic } \\
v(C=N) \\
\text { imidazole }\end{array}$ & $\begin{array}{c}v(\mathrm{C}-\mathrm{N}) \\
\text { imidazole }\end{array}$ & $\begin{array}{l}v(\mathrm{C}=\mathrm{O}) \\
\text { ester }\end{array}$ & $\begin{array}{c}v(\mathrm{C}-\mathrm{H}) \\
\text { aromatic }\end{array}$ \\
\hline 4 & & 1693 & 3207 & $\begin{array}{l}1631 \\
1577\end{array}$ & $\begin{array}{l}1286 \\
1313\end{array}$ & $\begin{array}{l}1728 \\
1712\end{array}$ & 3024 \\
\hline 5 & & 1685 & 3450 & $\begin{array}{c}1629 \\
1577,1596\end{array}$ & $\begin{array}{l}1286 \\
1311\end{array}$ & 1733 & 3020 \\
\hline 6 & & 1685 & 3200 & $\begin{array}{c}1629 \\
1577,1598\end{array}$ & $\begin{array}{l}1284 \\
1311\end{array}$ & 1710 & 3026 \\
\hline
\end{tabular}

Table (7) FT-IR spectral data $\left(\mathrm{cm}^{-1}\right)$ of oxepane benzimidazole isoprpylidene galactopyranose (7-9)

\begin{tabular}{|c|c|c|c|c|c|c|c|}
\hline $\begin{array}{c}\text { Comp. } \\
\text { No. }\end{array}$ & Compound Structure & $\begin{array}{c}v(C-N) \\
\text { imidazole } \\
v(C-N) \\
\text { nucleoside }\end{array}$ & $\begin{array}{l}v(\mathrm{C}=\mathbf{O}) \\
\text { ester }\end{array}$ & $\begin{array}{l}v(\mathrm{C}=\mathrm{O}) \\
\text { ketone }\end{array}$ & $\begin{array}{c}v(\mathrm{C}-\mathrm{O}) \\
\text { isopropylidine }\end{array}$ & $\begin{array}{c}v(\mathrm{C}-\mathrm{H}) \\
\text { aromatic }\end{array}$ & $\begin{array}{c}v(C=C) \\
\text { Aromatic } \\
v(C=N) \\
\text { imidazole }\end{array}$ \\
\hline 7 & & $\begin{array}{c}1288,1311 \\
1330\end{array}$ & 1700 & 1693 & 1074 & $\begin{array}{l}3053 \\
3026\end{array}$ & $\begin{array}{l}1631 \\
1577\end{array}$ \\
\hline 8 & & $\begin{array}{c}1286,1315 \\
1342\end{array}$ & 1700 & 1683 & 1100 & $\begin{array}{l}3024 \\
3060\end{array}$ & $\begin{array}{l}1631 \\
1577\end{array}$ \\
\hline 9 & & $\begin{array}{l}1286 \\
1311\end{array}$ & 1728 & 1689 & 1072 & 3064 & $\begin{array}{l}1629 \\
1577\end{array}$ \\
\hline
\end{tabular}


Table (8) FT-IR spectral data $\left(\mathrm{cm}^{-1}\right)$ of oxepane benzimidazole galactopyranose (10-12)

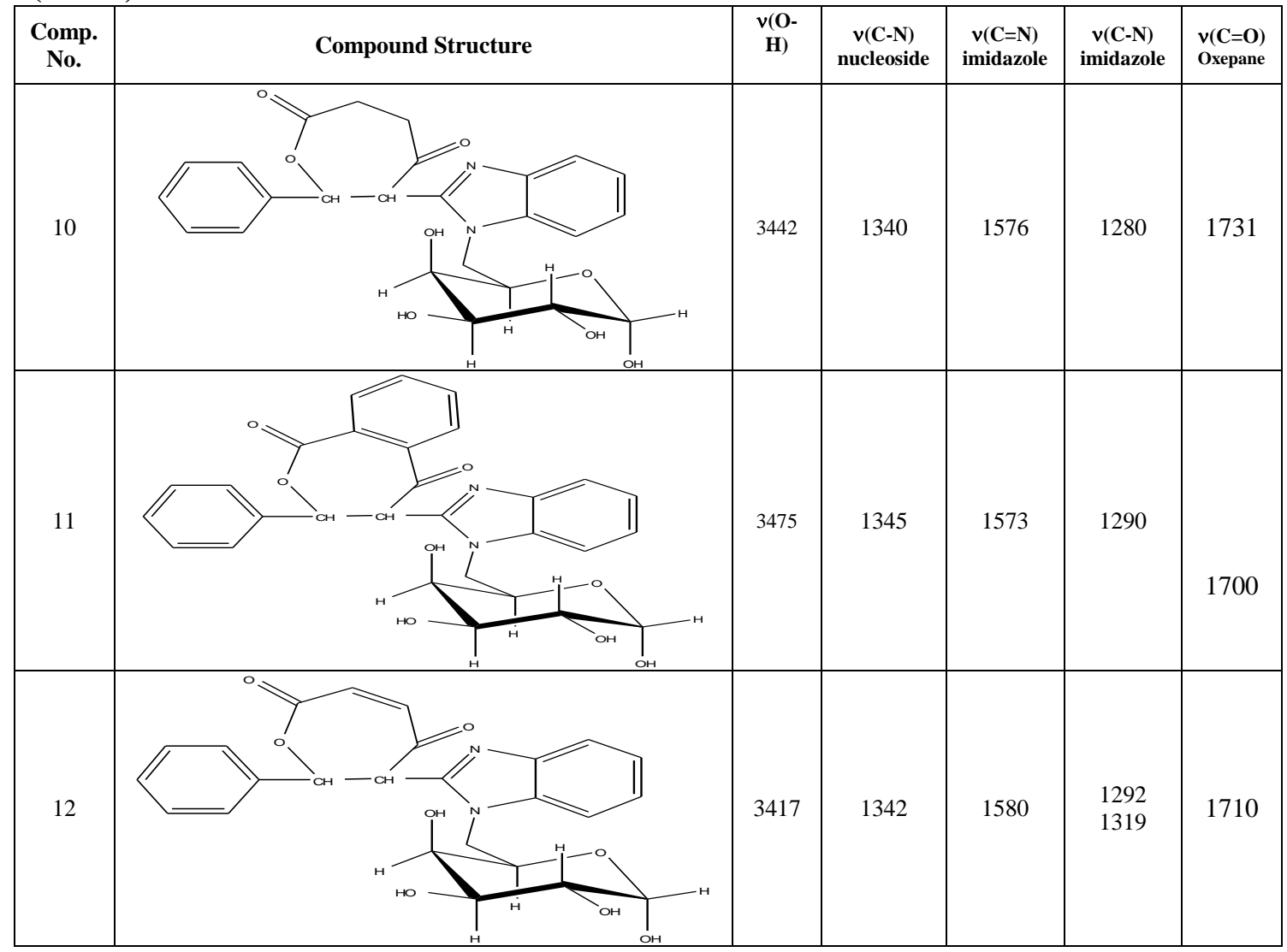

Table (9) physical properties of Substituted didehydrooxepane 4,7-diones (1416).

\begin{tabular}{|c|c|c|c|c|c|}
\hline $\begin{array}{l}\text { Comp. } \\
\text { No. }\end{array}$ & Compound Structure & Color & Yield \% & m.p $C^{0}$ & $\begin{array}{c}\text { Recrystallization } \\
\text { solvent }\end{array}$ \\
\hline 14 & & Gray & 72 & $102-104$ & THF \\
\hline 15 & & $\begin{array}{l}\text { Light } \\
\text { brown }\end{array}$ & 79 & $117-118$ & THF \\
\hline 16 & & Green & 70 & $170-172$ & THF \\
\hline
\end{tabular}


Table (10) physical properties of didehydrooxepane benzimidazole isopropylidene- D -galactopyranose (17-19) .

\begin{tabular}{|c|c|c|c|c|c|}
\hline $\begin{array}{l}\text { Comp. } \\
\text { No. }\end{array}$ & Compound Structure & Color & $\begin{array}{l}\text { Yield } \\
\%\end{array}$ & $\underset{\mathbf{C}^{0}}{\mathbf{m} . \mathbf{p}}$ & $\begin{array}{c}\text { Recrystallization } \\
\text { solvent }\end{array}$ \\
\hline 17 & & $\begin{array}{c}\text { Deep } \\
\text { brown }\end{array}$ & 52 & $\begin{array}{l}110- \\
112\end{array}$ & Chloroform \\
\hline 18 & & Brown & 60 & 96-98 & Chloroform \\
\hline 19 & & Gray & 47 & $\begin{array}{l}130- \\
132\end{array}$ & Chloroform \\
\hline
\end{tabular}

Table (11) physical properties of didehydrooxepane benzimidazole galactopyranose (20-22) .

\begin{tabular}{|c|c|c|c|c|c|}
\hline $\begin{array}{c}\text { Comp. } \\
\text { No. }\end{array}$ & Compound Structure & Color & Yield \% & m.p & $\begin{array}{c}\text { Recrystallization } \\
\text { solvent }\end{array}$ \\
\hline 20 & & Brown & 73 & $\begin{array}{l}225 \\
\text { dec }\end{array}$ & $\begin{array}{c}\text { Ethanol-ether } \\
(1: 1)\end{array}$ \\
\hline 21 & & $\begin{array}{l}\text { Light } \\
\text { brown }\end{array}$ & 79 & $\begin{array}{l}232 \\
\text { dec }\end{array}$ & Ethanol-ether(1:1) \\
\hline 22 & & $\begin{array}{l}\text { Light } \\
\text { brown }\end{array}$ & 71 & $\begin{array}{l}120 \\
\text { dec }\end{array}$ & Ethanol-ether(1:1) \\
\hline
\end{tabular}


Table (12) FT-IR spectral data $\left(\mathrm{cm}^{-1}\right)$ of didehydrooxepane benzimidazole (1416)

\begin{tabular}{|c|c|c|c|c|c|c|c|c|}
\hline $\begin{array}{c}\text { Comp. } \\
\text { No. }\end{array}$ & Compound Structure & $\begin{array}{c}v(\mathrm{~N}-\mathrm{H}) \\
\text { imidazole }\end{array}$ & $\begin{array}{c}v(\mathbf{C}=\mathbf{0}) \\
\text { ester }\end{array}$ & $\begin{array}{c}v(\mathrm{C}=\mathrm{O}) \\
\text { keton }\end{array}$ & $\begin{array}{c}v(\mathrm{C}=\mathrm{C}) \\
\text { Aromatic } \\
v(\mathrm{C}=\mathrm{N}) \\
\text { imidazole } \\
\end{array}$ & $\begin{array}{l}v(\mathrm{C}=\mathrm{C}) \\
\text { vinylic }\end{array}$ & $\begin{array}{c}v(\mathrm{C}-\mathrm{N}) \\
\text { imidazole }\end{array}$ & $\begin{array}{c}v(\mathrm{C}-\mathrm{H}) \\
\text { aromatic }\end{array}$ \\
\hline 14 & & 3444 & 1710 & 1685 & $\begin{array}{l}1631 \\
1570\end{array}$ & 1660 & 1319 & $\begin{array}{l}3024 \\
3056\end{array}$ \\
\hline 15 & & 3390 & 1700 & 1687 & $\begin{array}{l}1631 \\
1583\end{array}$ & 1665 & 1313 & $\begin{array}{l}3024 \\
3058\end{array}$ \\
\hline 16 & & 3467 & 1701 & 1683 & $\begin{array}{l}1631 \\
1570\end{array}$ & 1660 & 1315 & $\begin{array}{l}3024 \\
3060\end{array}$ \\
\hline
\end{tabular}

Table (13) FT-IR spectral data $\left(\mathrm{cm}^{-1}\right)$ of didehydrooxepane benzimidazole isoprpylidene galactopyranose (17-19)

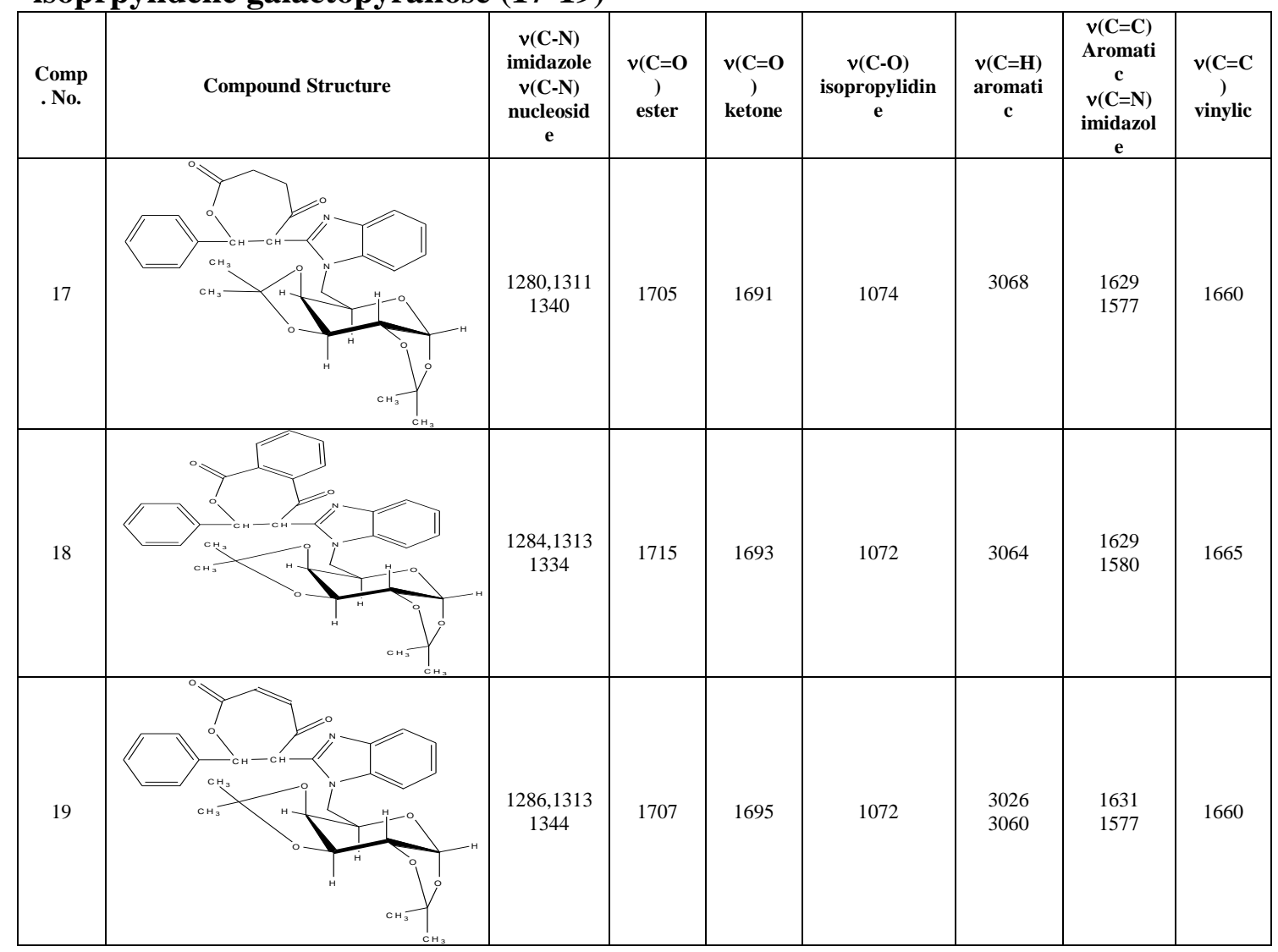


Table (14) FT-IR spectral data $\left(\mathrm{cm}^{-1}\right)$ of didehydrooxepane benzimidazole galactopyranose (20-22)

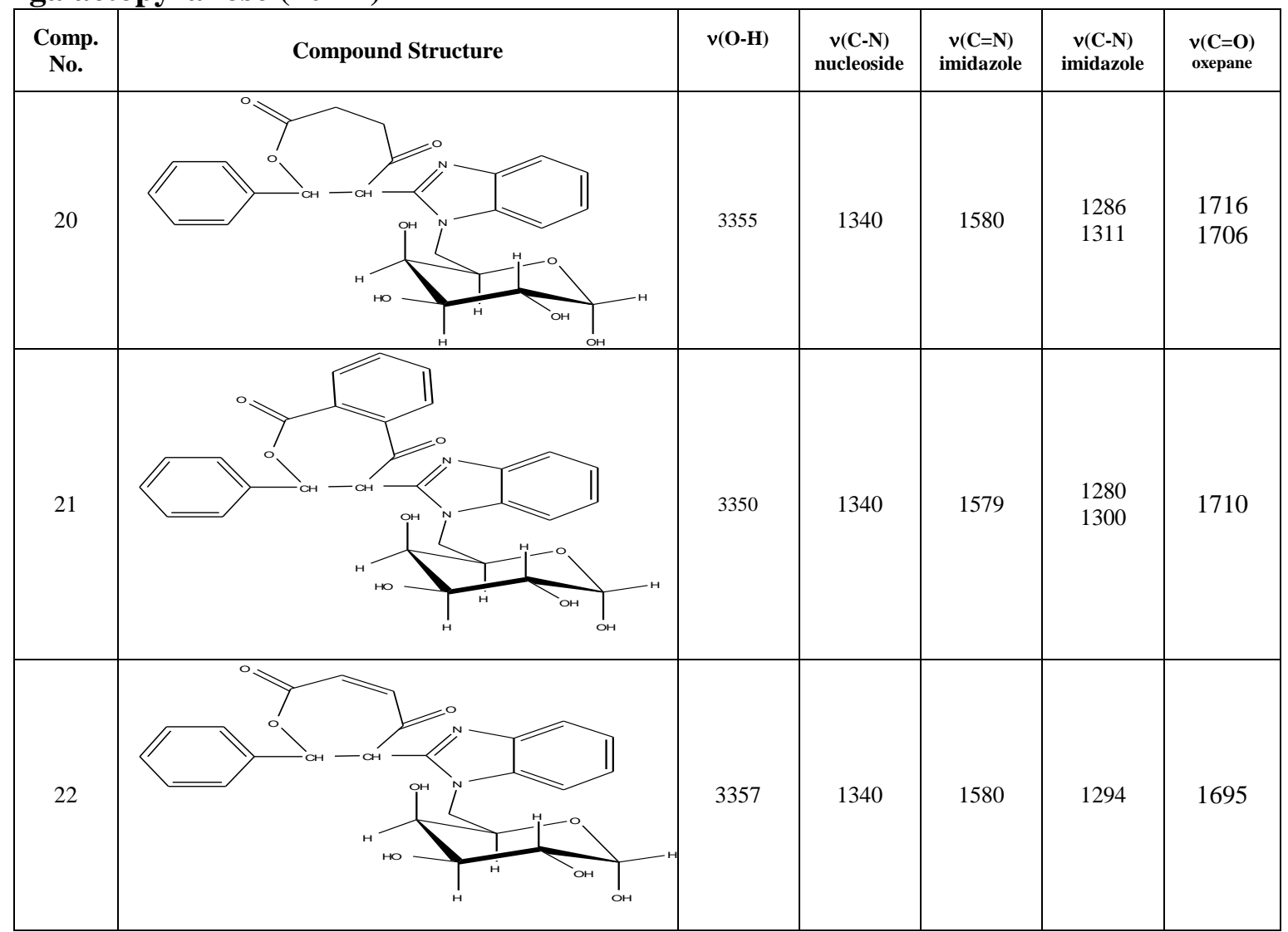

\section{References:}

[1] Pascal, G. 2011. Synthesis of oxepane Nucleosides by ring expansion and ring closing routes, master thesis, department of chemistry, McGill university Montreal ,QC, Canada.

[2] Hiryuki, Y.; Henki, R.; Tsuyoshi, K. 2012. A new Dibenz[ $b, e]$ oxepin -6 , 11 - dione , from a marine - derived fungus, beauveriabassiana TPU942, Mar. Drugs , 10(12):2691 - 2697.

[3] Vandr, E.A.;kravitz, E.J.;Harth, E.J. 2008. Approach to formation of multifunctional polyester particles in controlled nanoscopic dimensions, Am. Chem. Soc. 130 : 8706-8713.

[4] Passarell, R. J.; Spratt, D. E.; Vander, E. A. E.; Phillips, J. G. 2010. Targeted nanoparticles that deliver a sustained, specific release of paclitaxel to irradiated tumors, Cancer Res. 70(11) : 4550-9.
[5] Tej, B. Y.; Virendra, S. 2013. Iodine Promoted Simple Synthesis of benzimidazoleacyclonucleosides, IJC. 52B :1536- 1539.

[6] Ramanpreet, W.; Hedaitullah, M.; Syeda, F. N.; Khalid, I.; Lamba, H. 2011. Benzimidazole derivatives - an overview, IJRPC. 1(3): 565-574.

[7] Kurreck, J. 2003. Antisense technologies improvement through novel chemical modifications, Eur. J. Biochem. 270(8): 1628.

[8] Srivastav, N. C.; Shakya, N.; Bharanam, S.; Agrawal, A.; Kunimoto, D. Y.; Kumar, R. 2012. Discovery of novel 5-(ethyl or hydroxymethyl) analogs of 2'-'up' fluoro (or hydroxyl) pyrimidine nucleosides as a new class of Mycobacterium tuberculosis, Mycobacterium bovis and Mycobacterium avium inhibitors, Bioorg Med ChemLett. 20(13):1091. 
[9] Lin, J. B.; Shih, Y. C.; Chien, T. C. 2011. Study on the synthesis of 6alkylaminouridines via the nucleophilic aromatic substitution reactionof 6-cyanouridine derivatives, Tetrahedron let. 52(31): 3969 - 3972.

[10]Shih, Y. C.; Chien, T. C. 2011. Practical Synthesis of 6-Aryluridines via Palladium(II) Acetate Catalyzed Suzuki-Miyaura Cross-coupling Reaction, Tetrahedron, 67(21) : 3915- 3923.

[11]Jwad, R. S. 2011. Synthesis of 1Nonyl - 4- [ (6 - Deoxy - 1,2,3,4- di $-\mathrm{O}$ - isopropylidene - $\alpha-\mathrm{D}-$ Galactose $-6-$ yl ) Oxymethyl ], $\mathrm{H}$ - 1,2,3- Triazole via Click chemistry, JNUS. 14(1): $58-67$.

[12]Al-Mouamin, T. M. 2007. Synthesis of 2/ ,3/ -dideoxy-3/ ,3/ -gem-dicalkyl pyranonucleoside analogues. Ph.D. thesis, department of chemistry, college of science, Baghdad University, Iraq.

[13]Dubey, P. K.; kumar, R.; Grossert, J. S.; Hooper, D. L. 1999. A Facile and convenient method for the preparation of 2styrylbenzimidazoles, IJC. 38(B): 1211-1213.

[14]Ibraheem, H. A.; Salih, N. A. 2008. Synthesis and characterization of new fused heterocyclic compounds consisting of benzodiazepine, quinoxaline, benzimidazole andthiazole rings, B. S. J. 5(3): 440445.

[15]Collins, C. H.; Patricia, M. 1989. Microbiological methods, Lyne. $6^{\text {th }}$ ed, London, Butterworths.

[16]John Coates, 2000. Interpretation of infrared spectra, apractical approach, Encyclopedia of Analytical Chemistry R.A. Meyers (Ed.) pp. 10815-10837 John Wiley \& Sons Ltd, Chichester. 
تحضير بعض نظائر النيوكليوسيداتالجديدة المرتبطة بحلقة سباعية ودراسة فعاليتها البايلوجية

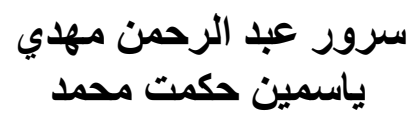

سرور عبد الرحمن مهدي

ياسمين حكمت محمد
ثناء مهدي المؤمن

عمر عبد اللطيف محمد

قسم الكيمياء، كلية العلوم، جامعة بغداد.

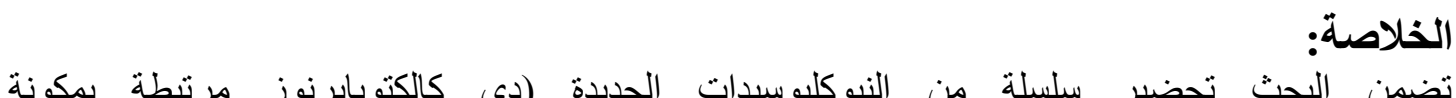

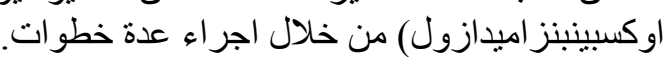

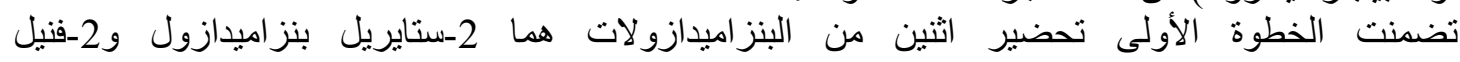

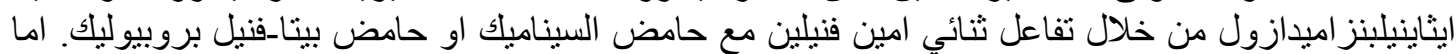

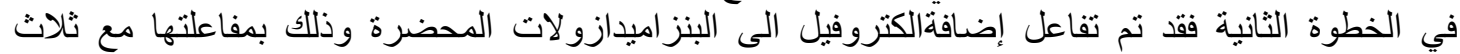

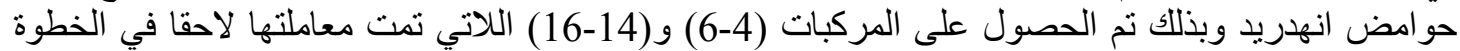

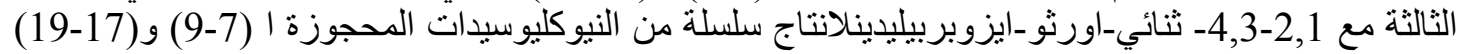

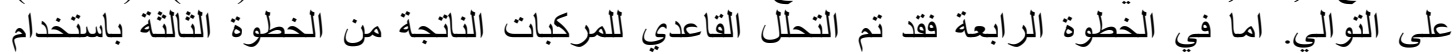

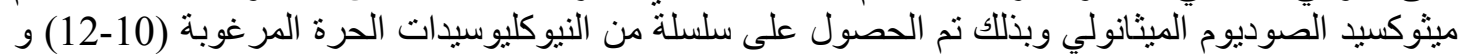

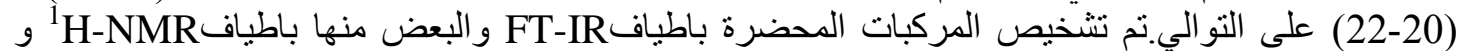
${ }^{13} \mathrm{C}-\mathrm{NMR}$

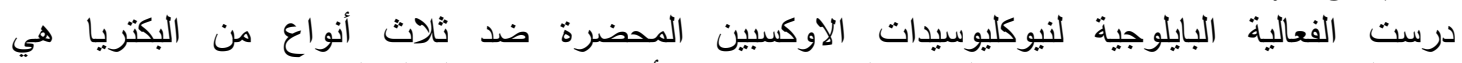

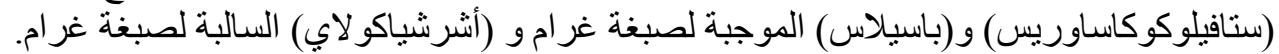

الكلمات المفتاحية: نيوكليو سيد ،اوكسبيننيو كليو سيد ، ايزوبروبيليدينكالكتوبايرنوز. 